\title{
Soft X-Ray Emission Studies of the Electronic Structure in Silicon Nanoclusters
}

\author{
T. van Buuren \\ L.N. Dinh \\ L.L. Chase \\ W.J. Siekhaus \\ I. Jimenez \\ L.J. Terminello \\ M. Grush \\ T.A. Callcott \\ J.A. Carlisle
}

This paper was prepared for submittal to the Materials Research Society 1996 Fall Meeting

Boston, MA

December 2-6, 1996

February 1997

This is a preprint of a paper intended for publication in a journal or proceedings. Since changes may be made before publication, this preprint is made available with the understanding that it will not be cited or reproduced without the permission of the author. 


\section{DISCLAIMER}

This document was prepared as an account of work sponsored by an agency of the United States Government. Neither the United States Government nor the University of California nor any of their employees, makes any warranty, express or implied, or assumes any legal liability or responsibility for the accuracy, completeness, or usefulness of any information, apparatus, product, or process

disclosed, or represents that its use would not infringe privately owned rights. Reference herein to any specific commercial product, process, or service by trade name, trademark, manufacturer, or otherwise, does not necessarily constitute or imply its endorsement, recommendation, or favoring by the United States Government or the University of California. The views and opinions of authors expressed herein do not necessarily state or reflect those of the United States Government or the University of California, and shall not be used for advertising or product endorsement purposes. 


\title{
SOFT X-RAY EMISSION STUDIES OF THE ELECTRONIC STRUCTURE IN SILICON NANOCLUSTERS
}

T. VAN BUUREN*, L. N. DINH*, L.L.CHASE*, W. J. SIEKHAUS*, I. JIMENEZ*, L.J. TERMINELLO*, M. GRUSH **, T.A. CALLCOTT**, J.A. CARLISLE $†$

* Chemistry and Materials Science Department, Lawrence Livermore National Laboratory, Livermore, CA , 94556

** Department of Physics, University of Tennessee, Knoxville, TN 37996

$\dagger$ Department of Physics, Virginia Commonwealth University, Richmond, VA 23284-2000

\begin{abstract}
Density of states changes in the valence and conduction band of silicon nanoclusters were monitored using soft x-ray emission and absorption spectroscopy as a function of cluster size. A progressive increase in the valence band edge toward lower energy is found for clusters with decreasing diameters. A similar but smaller shift is observed in the near-edge x-ray absorption data of the silicon nanoclusters.
\end{abstract}

\section{INTRODUCTION}

There are two main models for explaining the visible luminescence found in porous silicon. The first model " quantum confinement " is based on the idea that the restricted size of the nanometer scale silicon particles alters the band structure relative to bulk silicon [1]. In the competing model a surface layer or surface defect is responsible for the visible luminescence properties [2,3]. Recently it has been proposed that a combination of both models is needed to describe the optical properties found in porous silicon [4]. In order to shed more light on this matter, it seems useful to first examine more controllable systems, such as silicon nanocrystals with well defined sizes. In this paper, we show how Si nanoclusters with a narrow size dispersion can be synthesized in a well-controlled environment. The electronic structure of the silicon nanoclusters is then investigated using soft $\mathrm{x}$-ray emission (SXE) and $\mathrm{x}$-ray absorption spectroscopies. A prediction of the quantum confinement model is that the energies of the valence band (VB) and conduction band (CB) are shifted relative to the bands of bulk silicon. In order to overcome the charging and surface sensitivity problems of the electron spectroscopies we have used a method based on soft x-ray emission. SXE is explicitly a bulk-sensitive probe of the electronic structure. Photons penetrate many atomic layers deep into the material, as compared to a few atomic layers for electrons.

For the determination of the occupied valence electronic states we have measured the spectral distribution of the energy-resolved soft x-ray fluorescence (SXF) radiation generated in the transitions of the $\mathrm{Si}$ valence band electrons to the $\mathrm{Si} 2 \mathrm{p}_{3 / 2}$ core level. The core hole is selectively excited using monochromatized synchrotron radiation. Due to the photon in, photon out process the experiment is not affected by sample charging. With selective excitation it is furthermore feasible to separate the emission from $\mathrm{Si}$ atoms with and without oxygen neighbors, due to the chemical shift induced by the oxygen atoms.

Near-edge x-ray absorption fine structure spectroscopy (NEXAFS) measured with total photon yield can give complimentary information about the unoccupied states. This method is also bulk sensitive and is element specific since the incident x-ray photons excite electrons from selected core levels. The NEXAFS spectra were acquired by measuring the total $\mathrm{Si} \mathrm{L}_{2-3}$ emission yield with the same detector used for the fluorescence. SXF and NEXAFS measurements were performed on silicon nanoclusters synthesized in-situ on beamline 8.0 at the Advanced Light Sources. The beamline 8.0 and the fluorescence spectrometer are described in detail elsewhere [5].

\section{SAMPLE PREPARATION}

Silicon nanoclusters were synthesized by thermal vaporization of $\mathrm{Si}$ in an inert buffer gas [6]. The synthesis was conducted in a chamber with a base pressure of $<1 \times 10^{-8}$ Torr. Argon was leaked into the chamber up to the desired pressure. Pre-melted and outgassed Si inside a carbon boat, which was fitted inside a $\mathrm{W}$ basket, was resistively heated to a few hundred degrees 
above its melting point. Growth temperature was estimated with an optical pyrometer. The substrate used to collect the silicon nanocluster was a (111) oriented Ge wafer with a native oxide layer, mounted two inches directly above the evaporation boat. A shutter was placed between the evaporation boat and the substrate to control the Si arrival fluences. The size of the Si clusters was varied by increasing (or decreasing) the Si source temperature or the pressure of the Ar buffer gas. After synthesis, the Ar buffer gas was pumped out of the chamber and the as prepared silicon clusters were passivated by exposure to atomic hydrogen for 5 minutes. The atomic hydrogen source was molecular hydrogen at $1 \times 10^{-5}$ Torr passed through a very fine tungsten mesh at $2000^{\circ} \mathrm{C}$. After synthesis the samples were transferred into the ultra high vacuum (UHV) analysis chamber at the ALS beamline 8.0 without exposure to air.

To measure the size of the clusters a highly oriented pyrolitic graphite (HOPG) substrate was used as a witness sample for each deposition on Ge. The basal plane of graphite was chosen as a substrate because all the carbon bonds in this plane are satisfied, also the surface is atomically flat making it ideal for subsequent scanning tunneling microscope (STM) or atomic force microscope (AFM) measurements of clusters size. Characterization of the size and morphology of the gsynthesized material was done in situ using a STM and ex-situ using atomic force microscopy. In Fig. 1(a) we show an AFM image of silicon clusters deposited on HOPG by evaporation of $\mathrm{Si}$ at $1700^{\circ} \mathrm{C}$ in an argon buffer gas of 112 mTorr. We find the silicon nanoclusters gather at the step edges on the graphite surface or assemble into snowflake like superclusters. STM on one of the superclusters shows that each snowflake like structures is made up of many individual nanoclusters as reported earlier [4]. X-ray diffraction and high resolution transmission electron microscopy work

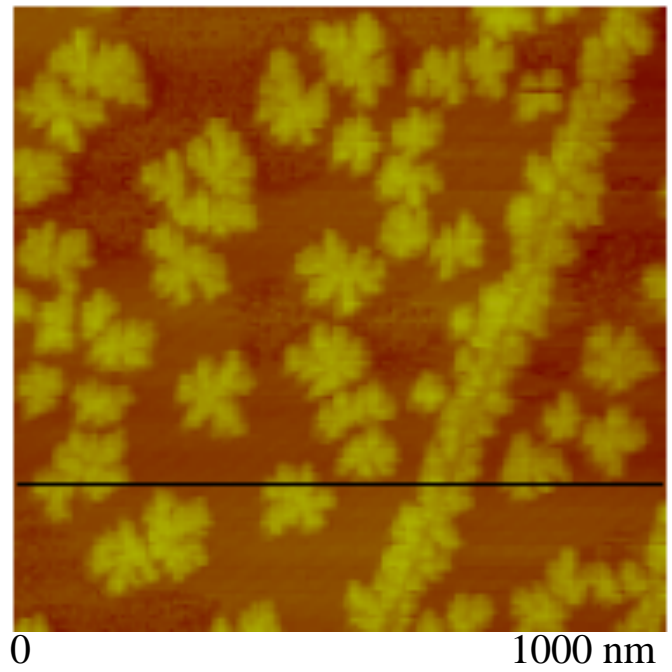

Fig.1(a)

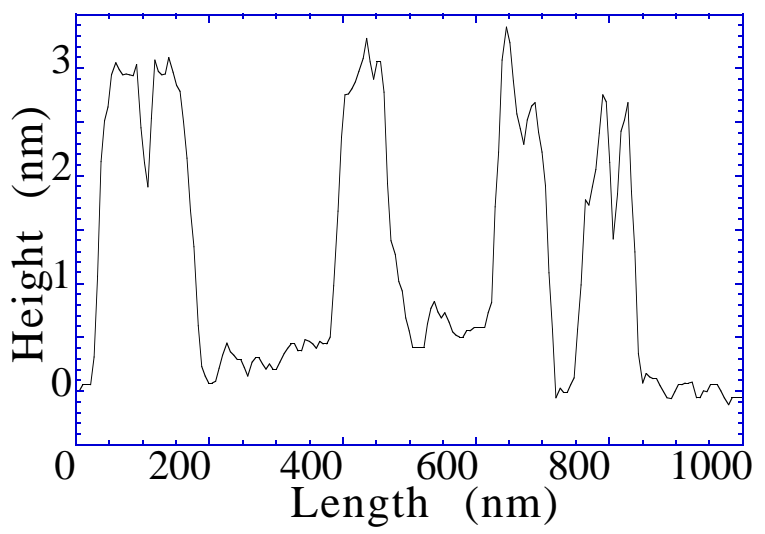

Fig.1(b)

Fig. 1(a). AFM image of silicon nanoclusters on the basal plane of HOPG formed by evaporation of $\mathrm{Si}$ at $1700^{\circ} \mathrm{C}$ in an argon buffer gas of $112 \mathrm{mTorr}$. Note how the nanoclusters gather at the step edges on the graphite surface or assemble into snowflake like superclusters. In Fig. 1(b) we shown the height cross-section along the black line on the AFM image.

has revealed that the silicon nanoclusters formed in this manner are crystalline and approximately spherical in shape. As shown in Fig. 1(b), the diameter of the nanoclusters is determined from the $\mathrm{z}$ scale of the AFM image, which is calibrated with a set of known standards. The average diameter of the clusters shown in Fig. 1 is $3 \mathrm{~nm}$, with distribution in size that is approximately $20 \%$ of the average size. The distribution shape has an asymmetric bell shape with a tail toward larger diameters. This is characteristic of the log-normal distribution found in ultrafine metal particles formed by evaporation in a reduced atmosphere of an inert gas [6]. Since the AFM is done in air, the silicon clusters will oxidize. 


\section{RESULTS}

Two Si cluster samples were grown by evaporation of $\mathrm{Si}$ at $1700^{\circ} \mathrm{C}$ in an argon buffer gas of 40 and 60 mTorr and deposited on a Ge substrate. AFM measurements after spectroscopic characterization show that the average diameter of the clusters is $1.6 \mathrm{~nm}$ in the $40 \mathrm{~m}$ Torr sample and $2.0 \mathrm{~nm}$ in the 60 mTorr sample. In Fig. 2 we show the NEXAFS spectra of the $\mathrm{L}_{2,3}$ edge absorption for bulk silicon and the two silicon nanocluster samples. The bulk silicon sample was oriented perpendicular to the incident photon flux and grazing incidence to the fluorescence detector to minimize the effects of self absorption in the total fluorescence yield NEXAFS spectra. The $\mathrm{L}_{2,3}$ -edge of the cluster samples is shifted to higher energy relative to the bulk silicon by $1.4 \mathrm{eV}$ for the $2.0 \mathrm{~nm}$ clusters and $2.2 \mathrm{eV}$ for the $1.6 \mathrm{~nm}$ clusters, in agreement with quantum confinement which raises the energy of the bottom of the conduction band as the nanocluster particle size is decreased. In addition the well defined double threshold behavior associated with the $0.6 \mathrm{eV}$ splitting of the $\mathrm{Si}$ $2 p$ core level is less pronounced in the L-edge of the nanoclusters. We also note that the onset of the absorption edge in the clusters is not as sharp as that in the bulk silicon. We attribute these features to a distribution of quantum shifts caused by the variation of particle size within the sample. This effect has been modeled by the absorption edge of bulk silicon shifted in energy to simulate the average quantum shift and broadened by convolution of a Gaussian determined by the size distribution measured in the AFM images [7].

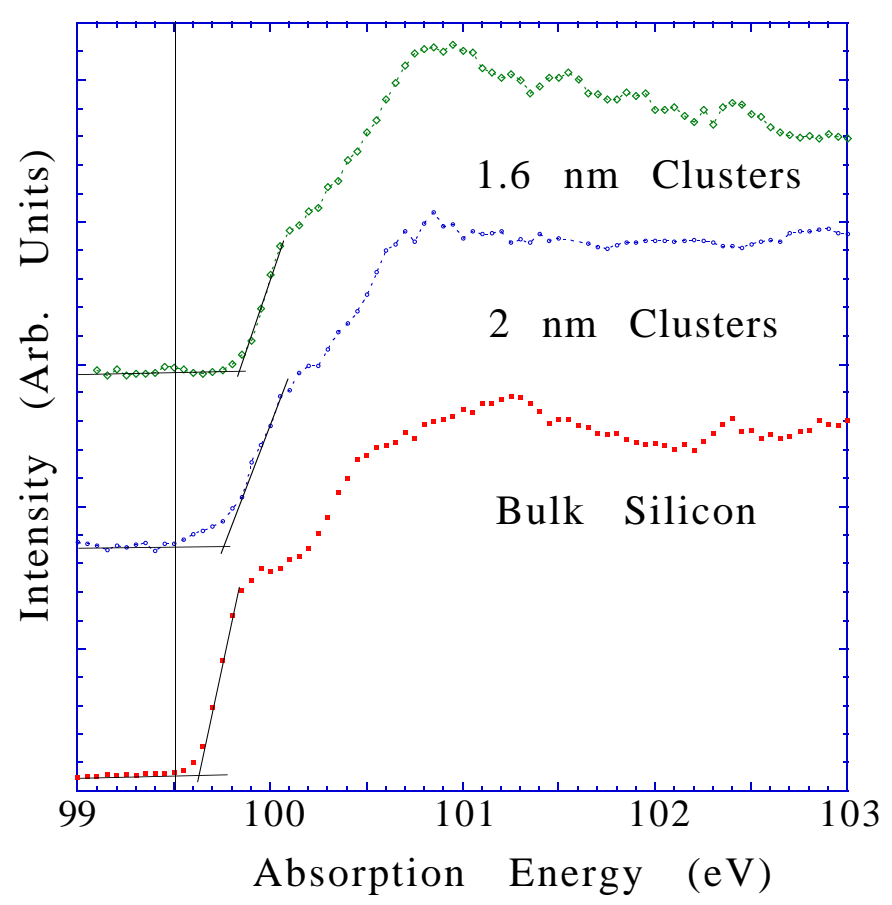

Fig. 2. Fluorescence yield $\mathrm{x}$-ray absorption spectra at the silicon $\mathrm{L}_{2,3}$ edges in bulk silicon, of silicon nanoclusters with an average size of $2.0 \mathrm{~nm}$ and $1.6 \mathrm{~nm}$. The solid line indicates the extrapolation of the $\mathrm{L}_{3}$ edge to baseline in order to determine the $\mathrm{CB}$ edge position.

We note that the L-edge absorption of the Si nanoclusters is very similar to that found in porous silicon [8,9]. Changes in the electronic structure of the VB were monitored by SXF spectroscopy on the same samples that there previously investigated by NEXAFS. In the SXF process a valence electron fills the core vacancy previously generated by the absorption of a photon. The generated fluorescence photon was analyzed in a spherical grating Rowland spectrometer. In all spectra presented the excitation energy was chosen to be below the $\mathrm{Si} \mathrm{L}_{2}$ absorption edge as determined from the NEXAFS spectra. Therefore, the fluorescence spectrum is generated by transitions from 
the valence band to the silicon $2 p_{3 / 2}$ core hole. The SXF technique has an advantage over photoemission because it is insensitive to sample charging and is a bulk probe due to the large photon mean free path ( 0.1 micron). By tuning the excitation energy below the Si L-edge absorption threshold of $\mathrm{SiO}_{\mathrm{x}}$ we selectively investigate the electronic structure of the $\mathrm{Si}$ nanocluster not any surface oxide or substrate feature.

In Fig. 3 we show the SXF spectra for bulk silicon and the same two cluster samples as shown in Fig. 2. The SXF spectra were excited at $100 \mathrm{eV}$ for the bulk silicon and the $1.6 \mathrm{~nm}$ clusters and $100.2 \mathrm{eV}$ for the $2 \mathrm{~nm}$ clusters. The bulk silicon SXF spectrum exhibits the three characteristic peaks, one at $89 \mathrm{eV}$ associated with low-lying 3s states, another due to a density of states (DOS) maximum at $91.5 \mathrm{eV}$ with strong s-p hybridization and a high DOS at $96 \mathrm{eV}$ which is dominated by p-type states [9-11]. The intense peak at approximately $100 \mathrm{eV}$ is due to reflected light from the undulator beamline into the spectrometer. The corresponding peak in the bulk Si spectrum is very weak as the reflectivity of the polished Si wafer is low in the non-specular position. Using these reflection peaks we reference the emission energy to the excitation energy and the NEXAFS spectra.

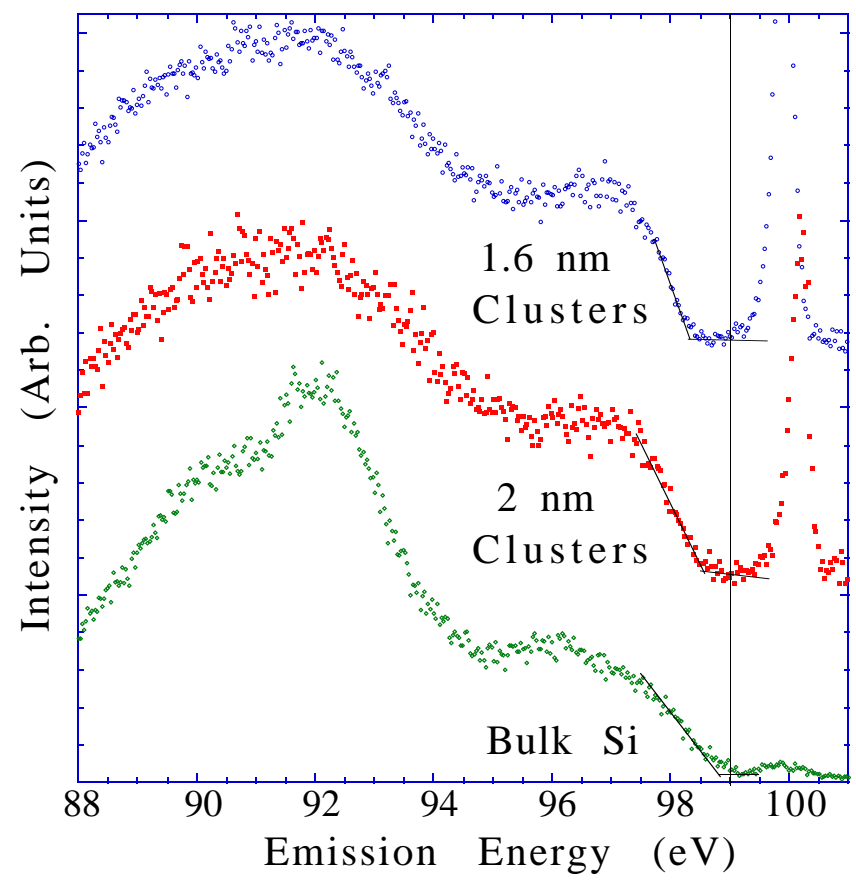

Fig. 3. The $\mathrm{L}_{3}$ emission spectrum of bulk silicon and the 2.0 and $1.6 \mathrm{~nm}$ diameter cluster samples. The excitation energy is $100 \mathrm{eV}$ for the bulk silicon and for the $1.6 \mathrm{~nm}$ clusters and $100.2 \mathrm{eV}$ for the $2 \mathrm{~nm}$ clusters. This corresponds to the $\mathrm{L}_{3}$ edge measured in the absorption spectra so the $\mathrm{L}_{2}$ edge is suppressed. The solid lines indicate the extrapolation of the high energy cut-off to the baseline in order to determine the VB edge position.

The overall appearance of the nanocluster spectra is different than that of the bulk silicon. In the nanocluster spectra the valence band is shifted to lower emission energy by $0.30 \mathrm{eV}$ and $0.51 \mathrm{eV}$ for the $2.0 \mathrm{~nm}$ and $1.6 \mathrm{~nm}$ clusters respectively. The VB edge is much sharper in the cluster samples than in the bulk Si opposite to what is observed in the X-ray absorption edge. Also the shape of the spectra is flattened from a peak like feature in the bulk Si to a plateau in the silicon nanoclusters. This effect has been observed in the SXF spectra of porous silicon and has been attributed to changes in the DOS at the VB edge due to confinement effects in the Si crystallites which alter the bulk electronic band structure [11].

The pronounced peak at $91.5 \mathrm{eV}$ is not observed in the SXF spectra for the silicon nanoclusters. In fact the spectra looks more like what is observed for amorphous silicon [10]. Yet 
X-ray diffraction and TEM studies of these clusters have shown they are crystalline in nature [4]. It is interesting to note that the peak at 91.5 is observed in the SXF spectra of hydrogenated porous silicon $[9,11]$.

It can be seen that the smaller silicon nanoclusters exhibit a larger VB shift as well as a larger conduction band shift. The VB shifts are larger than the shifts in the CB in accordance with theory and are similar to recent photoemission data on porous silicon although the ratio of the conduction band shift to valence band shift is slightly larger than that found in the photoemission study [8]. The observed band edge shifts are in qualitative agreement with the increasing luminescence blue shifts with decreasing particle size found in silicon nanoclusters with an oxide passivation [4].

In conclusion, we were able to investigate the unoccupied and occupied electronic states in silicon nanoclusters combining total fluorescence yield NEXAFS and selectively excited SXF spectroscopy. We observe shifts in the both the CB and VB edges indicating quantum size effects in the band structure of the nanoclusters. The onset of the absorption edge progressively broadens with increased confinement due to a distribution in cluster size. A steeper VB edge is observed in the nanoclusters as compared to bulk Si due to a change in the DOS at the VB edge.

\section{ACKNOWLEDGEMENTS}

We would like to thank Randy Hill for his technical assistance. This work was supported by the Director, Office of Energy Research, Office of Basic Energy Sciences, Chemical Sciences Division of the U.S. Department of Energy, LBNL under Contract No. DE-AC03-76SF00098 and LLNL under Contract No. W-7405-ENG-48, this work was done at the ALS, which is supported by the Department of Energy (Division of Materials Sciences and Division of Chemical Sciences of Basic Energy Sciences) under Contract No. DE-AC02-76CH0016.

\section{REFERENCES}

1. T. Canham, Appl. Phys. Lett. 57, 1046 (1990).

2. F. Koch, V. Petrova-Koch, and T. Muschik, J. Lumin. 57, 271 (1993).

3. S.M. Prokes, and O.J. Glembocki, Mater. Chem. Phys. 35, 1 (1993).

4. L.N. Dinh, L.L. Chase, M. Balooch, W.J. Siekhaus, F. Wooten, Phys. Rev. B 54, 5029 (1996).

5. J.J. Jia, T.A. Callcott, J. Yurkas, A.W. Ellis, F.J. Himpsel, M.G. Samant, D.L. Ederer, J.A. Carlisle, E.A. Hudson, L.J. Terminello, D.K. Shuh, and R.C.C. Perera, Rev. Sci. Instrum. 66, 1394 (1995).

6. C. G. Granqvist, R.A. Buhrman, J. Appl. Phys. 47, 2200 (1976).

7. T. van Buuren, L.N. Dinh, L.L. Chase to be published.

8. T. van Buuren, T. Tiedje, J.R. Dahn, and B.M. Way, Appl. Phys. Lett. 63, 2911 (1993).

9. S. Eisebitt, J. Luning, J.-E. Rubensson, T. van Buuren, S.N. Patitsas, T. Tiedje, M. Berger, R. Arens-Fisher, S. Frohnhoff and W. Eberhardt, Solid State Comm. 97, 549 (1996).

10. K.E. Miyano, D.L. Ederer, T.A. Callcott, W.L. O’Brien, J.J. Jia, L.Zhou, Q.-Y. Dong, Y.Ma, J.C. Woicik and D.R. Mueller, Phys. Rev B 48,1918 (1993).

11. S. Eisibitt, S.N. Patitsas, T. Tiedje, T. van Buuren, J. Luning, J.-E. Rubensson and W. Eberhardt, submitted to Euro. Phys. Lett. 


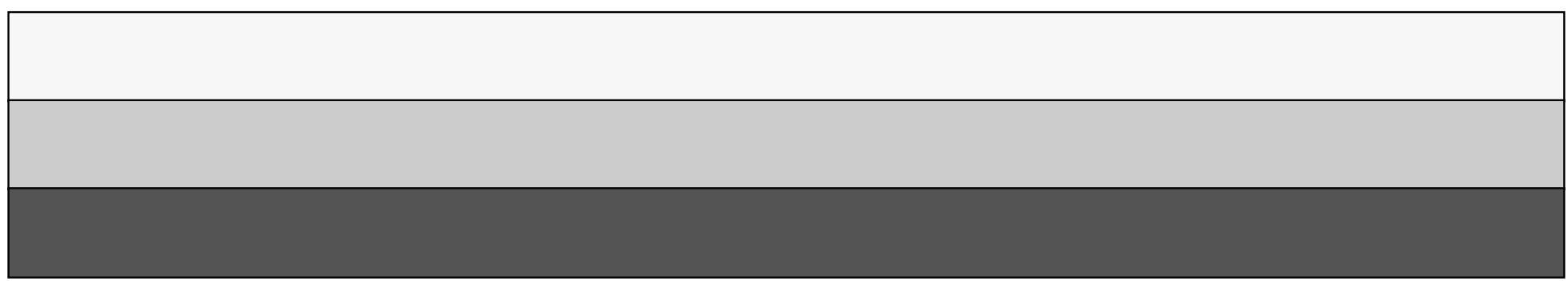

